\title{
Carotenoid pigment levels in Staphylococcus aureus and sensitivity to oleic acid
}

\author{
Z. XIONG and F. A. KAPRAL \\ Department of Medical Microbiology and Immunology, The Ohio State University, Columbus, OH 43210, USA
}

\begin{abstract}
Summary. Staphylococcus aureus mutants lacking pigment, or expressing only low levels of pigment, were more sensitive to oleic acid than were the parent strain and mutants making more pigment than the parent. One class of mutants (colour index 5), although possessing significant levels of pigment, were nevertheless quite sensitive to oleic acid. This suggested that only certain carotenoids in the biosynthetic pathway were capable of imparting resistance to fatty acids. The phenotypic expression of pigment also affected the sensitivity of a strain to oleic acid. The parent $S$. aureus strain $18 \mathrm{Z}$, when grown to express its maximal pigment potential, was more resistant to oleic acid than when it was grown to express minimal levels of pigment.
\end{abstract}

\section{Introduction}

Pigmentation in Staphylococcus aureus can be attributed to the possession of a group of triterpenoid carotenoids, but only recently have their biosynthetic pathways been elucidated. ${ }^{1-3}$

Carotenoid production among other bacteria is quite uncommon ${ }^{4}$ In the case of Sarcina lutea, the carotenoid pigments seem to afford some protection against intracellular killing by phagocytes, possibly by quenching singlet oxygen within the phagosome. ${ }^{5}$ In another study comparing the sensitivity of pigmented and non-pigmented strains of $S$. aureus to singlet oxygen, the pigmented strains were killed more slowly. ${ }^{6}$ Unfortunately, the strains being compared were unrelated and, therefore, could have differed in other respects besides carotenoid production.

Studies in our laboratory over the past decade have revealed that the host controls the growth and survival of $S$. aureus within abscesses by the production of two types of bactericidal lipid. ${ }^{7-11}$ On this basis we have considered the possibility that the carotenoid pigments might affect the sensitivity of $S$. aureus to these lipids. Oleic acid was selected for these studies because it is the predominant bactericidal fatty acid present in staphylococcal abscesses. ${ }^{9}$

\section{Materials and methods}

\section{Bacterial strain}

S. aureus strain $18 \mathrm{Z}$ was selected since it has served as a prototype strain for most of our previous studies. ${ }^{10}$

Received 26 June 1991; revised version accepted 23 Dec. 1991.
This strain is typical of $S$. aureus isolates of human origin, producing the $\alpha$ and $\delta$ toxins, coagulase and clumping factor.

\section{Media}

Trypticase-yeast extract broth (TYB) consisted of trypticase peptone (BBL) $1.7 \%$, yeast extract (Difco) $1 \%$, sodium chloride $0.5 \%$ and dibasic potassium phosphate $0.25 \%$.

Carotenoid expression medium (CEM) was composed of TYB to which was added glycerol monoacetate $0.4 \%$ and Bacto Agar $1 \%$. The $\mathrm{pH}$ was adjusted to 7.9 before autoclaving. Incubation was for $48 \mathrm{~h}$ at $37^{\circ} \mathrm{C}$ and a further $24 \mathrm{~h}$ at room temperature $\left(22-23^{\circ} \mathrm{C}\right)$.

\section{Isolation of carotenoid mutants}

Following the approach of Marshall and Wilmoth, ${ }^{2}$ the parental strain, S. aureus $18 \mathrm{Z}$, was grown at $37^{\circ} \mathrm{C}$ for $18 \mathrm{~h}$ in TYB. Mutagenesis was induced by adding $0.08 \mathrm{ml}$ of ethylmethane sulphonate to $1 \mathrm{ml}$ of whole culture and incubating the mixture at $37^{\circ} \mathrm{C}$ for $20 \mathrm{~min}$; $8 \mathrm{ml}$ of saline was added and the mixture was centrifuged to sediment the organisms. The cocci were suspended in $10 \mathrm{ml}$ of TYB and incubated at $37^{\circ} \mathrm{C}$ for $4 \mathrm{~h}$. The suspension was diluted and samples were plated on CEM and incubated under the previously stated conditions. Any colonies expressing levels of pigmentation that were less than, or greater than, the parent strain were isolated. The pigment production of each isolate (relative to the parent) was subsequently verified by again streaking the organisms on CEM. To ensure that the isolates were, in fact, mutants and not 
contaminants, each isolate was examined for Gram's stain characteristics and the production of $\alpha, \beta$ and $\delta$ toxins, coagulase and clumping factor. Only those isolates possessing the same properties as the parent strain were selected for further study.

After a number of carotenoid mutants had been isolated, a colour scale (colour index) to signify the colour and intensity of pigment produced was established according to the method of Marshall and Wilmoth. ${ }^{2}$ The colour index ranged from 0 to 9 and the designations are described in the table. Each mutant was assigned a colour index according to the maximum pigment produced on CEM under standard conditions. The reversion rate of one mutant was 1 in 200, two were $c .1$ in 2000 and the remainder were $<1$ in 10000 .

\section{Bactericidal assay}

Measurement of the sensitivity of the parental strain and mutants therefrom to oleic acid was performed by previously described procedures. ${ }^{8}$ Briefly, an oleic acid stock solution in ethanol was diluted 1 in 10 in diluent ( $2 \mathrm{M} \mathrm{NaCl}$ with $2 \mathrm{mM}$ EDTA) just before use. Further serial two-fold dilutions of the emulsion in diluent were made and a standard number of bacteria ( $500 \mathrm{cfu})$ being tested was added to each dilution. After incubating at $37^{\circ} \mathrm{C}$ for $1 \mathrm{~h}$ in a water bath with shaking, $0.5 \mathrm{ml}$ samples of each tube were plated on trypticase soy agar to enumerate the number of surviving cocci. Several control tubes (without oleic acid) were included in each assay. Each assay was done in duplicate. The LD50 was determined by the method of probits ${ }^{12}$ and was expressed as $\mu \mathrm{g} / \mathrm{ml}$.

\section{Results}

\section{Factors influencing pigment expression}

It is well known that pigment production by $S$. aureus is influenced significantly by cultural con-

Table. Relative sensitivity of $S$. aureus pigmentation mutants to oleic acid

\begin{tabular}{llcc}
\hline $\begin{array}{c}\text { Colour } \\
\text { index }\end{array}$ & Description & $\begin{array}{c}\text { Number } \\
\text { of mutants } \\
\text { isolated* } \\
(\%)\end{array}$ & $\begin{array}{c}\text { ED50 of } \\
\text { oleic acid } \\
(\mu \mathrm{g} / \mathrm{ml})\end{array}$ \\
\hline 0 & White & $39(33 \cdot 9)$ & 14 \\
1 & Cream & $17(14 \cdot 8)$ & 17 \\
2 & Yellow & $12(10 \cdot 4)$ & 19 \\
3 & Greenish-yellow & $11(9 \cdot 6)$ & 32 \\
4 & Light yellow-orange & $8(7 \cdot 0)$ & $>50$ \\
5 & Yellow-orange & $19(16 \cdot 5)$ & 10 \\
6 & (Parent) Orange & $7 \ldots$ & $>50$ \\
7 & Dark orange & $7(6 \cdot 1)$ & $>50$ \\
8 & Dark yellow-orange & $1(0.9)$ & $>50$ \\
9 & Salmon & $1(0.9)$ & $>50$ \\
\hline
\end{tabular}

*Total number of isolates was 115 . ditions. To compare the level of pigment in the parental strain and the derived mutants, it was necessary to determine conditions which would maximise pigment production. Therefore, a number of parameters were evaluated for their impact on carotenoid production. These included the base medium (trypticase soy broth, TYB, nutrient broth), the type of agar (agar USP, Bacto agar, purified agar, ionagar, agarose), agar concentration $(0.6-2.0 \%)$, glycerol monoacetate concentration $(0 \cdot 2-6 \%)$, $\mathrm{pH}(6 \cdot 5-7 \cdot 9)$, concentration of $\mathrm{CO}_{2}$ in the atmosphere during incubation (0-20\%), and exposure to light. Of these, the base medium, type of agar, concentration of agar, concentration of glycerol monoacetate, and $\mathrm{pH}$ had a significant impact on pigment production, but $\mathrm{CO}_{2}$ and light did not. The formulation finally selected (CEM) optimised the aforementioned parameters and consistently permitted maximum carotenoid expression when incubated under standard conditions.

\section{Isolation of pigmentation mutants}

After induction of mutagenesis, cultures were plated on CEM and colonies differing from the parent with respect to pigment intensity or colour were isolated. Once a sufficient number of mutants had been accumulated, a colour scale (colour index) was established similar to that used by Marshall and Wilmoth. ${ }^{2}$ Thereafter, representatives of each colour index were streaked side by side on the same plate of CEM and served as a standard with which all other mutants were compared. A fresh standard plate was prepared every time additional mutants were isolated. All mutants were re-plated on CEM and compared to a standard plate to verify the colour index to which it was assigned.

A total of 115 confirmed mutants was isolated and they were each assigned a colour index as shown in the table. Those with colour indices $0-5$ produced less pigment than the parental strain (colour index 6), and those with colour indices 7-9 produced more pigment.

\section{Sensitivity of pigmentation mutants to oleic acid}

Representatives of each colour index were assayed for their sensitivity to oleic acid. Mutants with colour indices of $0-3$ were demonstrably more sensitive to the fatty acid than was the parent strain, whereas mutants with colour indices 4 and 7-9 were just as resistant as the parent (table). Mutants with colour index 5, although obviously well pigmented, were nevertheless very sensitive to oleic acid.

\section{Phenotypic expression of pigment}

Since mutants possessing little or no pigment were found to be more sensitive to oleic acid, and since the level of pigment which may be expressed even by a fully-pigmented strain is governed by the cultural conditions, we examined the sensitivity of the parental 
strain when expressing different degrees of pigmentation. S. aureus $18 \mathrm{Z}$ was grown either on CEM to express maximal amounts of pigment (colour index 6) or in TYB to express minimal amounts of pigment (colour index 1 ). The strain was decidedly more sensitive to oleic acid (LD50 5.6 $\mu \mathrm{g} / \mathrm{ml}$ ) when it possessed low levels of pigment than when it was expressing the maximal amount of pigment (LD50 > $50 \mu \mathrm{g} / \mathrm{ml}$ ). This response was totally reversible.

\section{Discussion}

The bactericidal lipids produced in abscesses consist of a pool of long-chain unsaturated free fatty acids, of which oleic acid is the most prominent, and a pool of monoglycerides, of which the 2-monoglycerides are the most important.

It has been recognised for some time that certain lipids such as monoglycerides and free fatty acids, when incorporated into growth media, can stimulate pigment production by $S$. aureus. ${ }^{13,14}$ These are the same types of lipid as those produced by the host to control the growth and survival of the staphylococci within focal lesions. ${ }^{8,9}$ However, a notable difference is that the fatty acids produced by the host (either as free fatty acids or as the fatty acid moiety in the monoglycerides) are long-chain, unsaturated and bactericidal, whereas those generally used to stimulate pigment production are short-chain, saturated and non-toxic.

In the present study, mutants with colour indices of $0-3$, which could make little or no pigment, were more sensitive to oleic acid than was the parental strain (table). On the other hand, mutants with colour indices of 4 and 7-9, that made ample amounts of pigment, did not differ from the parental strain in their sensitivity to the fatty acid. However, mutants assigned to colour index 5, although significantly pigmented, were quite sensitive to oleic acid. The observed level of pigmentation under optimal conditions would pre-

\section{References}

1. Marshall JH, Wilmoth GJ. Pigments of Staphylococcus aureus, a series of triterpenoid carotenoids. J Bacteriol 1981; 147: 900-913.

2. Marshall JH, Wilmoth GJ. Proposed pathway of triterpenoid carotenoid biosynthesis in Staphylococcus aureus: evidence from a study of mutants. J Bacteriol 1981; 147: 914 919.

3. Taylor RF, Davies BH. The triterpenoid carotenoids and related terpenoids in Staphylococcus aureus 209P. J Biochem Cell Biol 1982; 61 : 892-905.

4. Taylor RF. Bacterial triterpenoids. Microbiol Rev 1984; 48: 181-198.

5. Krinsky NI. Singlet excited oxygen as a mediator of the antibacterial action of leukocytes. Science 1974; 186: 363-365.

6. Dahl TA, Midden WR, Hartman PE. Comparison of killing of gram-negative and gram-positive bacteria by pure singlet oxygen. J Bacteriol 1989; 171 : 2188-2194.

7. Dye ES, Kapral FA. Survival of Staphylococcus aureus in sumably be the result of individual contributions made by all eight or nine carotenoids in the biosynthetic pathway. ${ }^{2,3}$ Since the presence and level of each carotenoid in the pathway was not evaluated for each colour index, the possibility remains that only certain carotenoids actually affect sensitivity to the fatty acids and thus the overall level of pigmentation may not be a true indicator of a strain's degree of resistance to the lipid. An answer to this question would require a detailed analysis of each class of mutant to determine which carotenoids are lacking or produced in limiting amounts.

It could be argued that the mutants were, in fact, pleiotropic mutants and that the observed changes in sensitivity to oleic acid were due to some factor unrelated to the carotenoids. However, it seems unlikely that the putative factor would be simultaneously lost in so many carotenoid mutants. Furthermore, such a situation would not easily explain the phenotypic changes linking carotenoid level with sensitivity to the fatty acid.

It remains to be determined also whether mutants deficient in carotenoid production differ in their sensitivity to monoglycerides. It does not follow necessarily that changes in sensitivity to one lipid are accompanied by similar changes to the other. The bactericidal fatty acids and monoglycerides appear to act upon staphylococci independently.

It is likely that the fatty acids kill staphylococcal cells by an action upon their cytoplasmic membranes. There is now considerable evidence supporting the view that carotenoids, upon incorporation into the membrane, can act as membrane stabilisers ${ }^{4}$ resulting in bilayer condensation, increased rigidity, and reduced fluidity. By such means, staphylococci with high carotenoid levels might be afforded greater protection from the action of fatty acids than cocci with little or no pigment.

This study was supported by Public Health Service grant AI-19879 from the National Institute of Allergy and Infectious Diseases.

intraperitoneal abscesses. $J$ Med Microbiol 1981; 14 185-194.

8. Dye ES, Kapral FA. Partial characterization of a bactericidal system in staphylococcal abscesses. Infect Immun 1980; 30: 198-203.

9. Dye ES, Kapral FA. Characterization of a bactericidal lipid developing within staphylococcal abscesses. Infect Immun 1981; 32: 98-104.

10. Kapral FA, Godwin JR, Dye ES. Formation of intraperitoneal abscesses by Staphylococcus aureus. Infect Immun 1980; 30: 204-211.

11. Kapral FA, Mortensen JE. Mechanisms of resistance to staphylocidal fatty acids in abscesses. In: Jeljaszewicz J (ed) The staphylococci. Zentralbl Bakteriol Suppl 14. Stuttgart, Gustav Fischer Verlag. 1985: 507-509.

12. Finney DJ. Probit analysis, 3 rd edn. Cambridge, The University Press. 1971.

13. Willis AT, Turner GC. Staphylococcal lipolysis and pigmentation. J Pathol Bacteriol 1962; 84: 337-347.

14. Willis AT, O'Connor JJ, Smith JA. Colonial pigmentation of Staphylococcus aureus. J Pathol Bacteriol 1966; 92: 97-106. 